\title{
Envelhecimento ativo: representações sociais dos profissionais de saúde das Unidades de Referência à Saúde do Idoso*
}

\author{
Active ageing: social representations of health professionals in Elderly Health Reference Units \\ Envejecimiento activo: representaciones sociales de profesionales de la \\ salud en las Unidades de Referencia para la Salud de los Ancianos
}

Como citar este artigo:

Sciama DS, Goulart RMM, Villela VHL. Active ageing: social representations of health professionals in Elderly Health Reference Units. Rev Esc Enferm USP. 2020;54:e03605. doi: https://doi.org/10.1590/S1980-220X2018056503605

\section{Debora Sipukow Sciama ${ }^{1}$ \\ Rita Maria Monteiro Goulart ${ }^{2}$ \\ Vera Helena Lessa Villela ${ }^{1}$}

* Extraído da tese: "O cuidado necessário: Representações sociais dos profissionais de saúde das Uninidades de Referência à Saúde do idoso do município de São Paulo", Universidade São Judas Tadeu, 2017.

${ }^{1}$ Secretaria Municipal da Saúde, Coordenadoria de Vigilância em Saúde, Divisão de Vigilância Epidemiológica, Núcleo de Doenças e Agravos Não Transmissíveis, São Paulo, SP, Brasil.

${ }^{2}$ Universidade São Judas Tadeu, Programa de Pós-graduação em Ciências do Envelhecimento, São Paulo, SP, Brasil.

\begin{abstract}
Objective: To identify social representations of health professionals providing elderly care in seven Elderly Health Reference Units in São Paulo city regarding health necessities, their role in care and promotion of active ageing. Method: Qualitative research, based on directives from the document "Active ageing: a policy framework" by the World Health Organization. The Discourse of the Collective Subject is employed to systematize social representations, whose central ideas were categorized using the QualiQuantiSoft ${ }^{\circledR}$ program. Four reference situations were elaborated and the one related to iatrogenesis and elder vulnerability was selected. Results: Twenty-nine professionals took part in this study (sixteen physicians, seven nutritionists and six nurses). Seven categories emerged from the central ideas, with higher frequency for medical conduct disagreement on referring the patient to the Basic Health Unit and the polypharmacy issue. Conclusion: Discourse by professionals point out the necessity of creating care that overcomes the fragmentation of the work process.
\end{abstract}

DESCRIPTORS

Aging; Aged; Public Policies; Health Personnel; Health Services for the Aged. 


\section{INTRODUÇÃO}

Discutir a promoção da saúde do idoso significa pensar a Promoção da Saúde como um caminho possível para a construção das políticas públicas. Que espaço é esse das Unidades de Referência à Saúde do Idoso (URSIs), em que se articulam envelhecimento ativo e promoção da saúde, e quais as concepções que lhe dão sustentação?

O Envelhecimento Ativo, desenvolvido pela Unidade de Envelhecimento e Curso de Vida da Organização Mundial da Saúde (OMS), é uma Política de Saúde que oferece informações e subsidia a formulação de planos de ação voltados para promover um envelhecimento saudável e ativo na rede de atenção ${ }^{(1)}$.

A OMS adotou o termo "envelhecimento ativo" para expressar o processo de conquista de oportunidades contínuas em seus três pilares: saúde, participação e segurança, com o objetivo de melhorar a qualidade de vida na medida em que as pessoas ficam mais velhas. No documento "Envelhecimento ativo: uma política de saúde"(1), o pilar saúde apresenta as diretrizes: "1.1. Prevenir e reduzir a carga de deficiências em excesso, doenças crônicas e mortalidade prematura; 1.2. Reduzir os fatores de risco associados às principais doenças e aumentar os fatores que protegem a saúde durante a vida; 1.3. Desenvolver um contínuo de serviços sociais e de saúde acessíveis, baratos, de alta qualidade e adequados para a terceira idade, que aborde as necessidades e os direitos de homens e mulheres em processo de envelhecimento; 1.4. Fornecer treinamento e educação para cuidadores".

Apesar dos avanços alcançados por esse documento, ainda existem carências quanto à implementação desses pilares. Em particular, a recente crise social e econômica impactou as políticas sociais e as instituições públicas, que sofreram cortes significativos. Isso não permitiu compensar a diminuição de rendas familiares, a perda de seguro social e a instabilidade no recebimento de pensões de muitos cidadãos, inclusive idosos. Esse cenário reforça a importância de se construírem planos que incorporem os princípios do envelhecimento ativo ${ }^{(2)}$.

Os profissionais de saúde envolvidos no cuidado da população idosa, segundo o proposto nas políticas de saúde, devem desenvolver uma atenção integral e interdisciplinar com base na clínica ampliada. Porém, há evidências de que tal proposta vem sendo implementada com restrições em razão da insuficiência na formação dos profissionais e pela forma de organização do processo de trabalho na saúde. A formação em saúde para atenção primária ainda se dá de forma fragmentada, especializada e com base num modelo biológico, mesmo com todos os avanços obtidos ${ }^{(3)}$.

As URSIs são serviços de saúde especializados no atendimento da população idosa em sua área de abrangência. Inseridas no nível secundário de atenção, desenvolvem ações de prevenção, promoção e proteção à saúde, bem como ações educativas, sempre com perfil interdisciplinar e intersetorial, indo ao encontro do que se preconiza de maneira mais abrangente na Política Nacional de Promoção da Saúde ${ }^{(4)}$.

Compete aos profissionais das URSIs avaliar todos os aspectos de queixa do paciente, como os psicossociais e comorbidades, bem como entender em que fase do processo de envelhecimento o idoso se encontra (robusto, pré-frágil ou frágil) para posterior tomada de conduta.

Para avaliar a interpretação dos profissionais das URSIs quanto ao cuidado da população idosa, optou-se por adotar a Teoria das Representações Sociais. Esta, na sua abordagem estrutural, considera o processo cognitivo e, consequentemente, além de abranger teoria e métodos de trabalho, acata o impacto e a influência dos fatores sociais e da própria linguagem escolhida sobre a caracterização, a interação e a avaliação dessas representações ${ }^{(5)}$. Nessa direção, acredita-se na possibilidade da compreensão do processo do cuidado, aproximando a subjetividade dos entrevistados às suas representações sociais sobre o trabalho na área da saúde e o envelhecimento na sociedade contemporânea. Dessa forma, este estudo teve o objetivo de identificar as representações sociais de profissionais que atendem idosos em todas as sete URSIs do município de São Paulo quanto às necessidades em saúde, bem como seu papel no cuidado e na promoção do envelhecimento ativo.

\section{MÉTODO}

\section{TIPO DE ESTUdo}

Trata-se de um estudo qualitativo, transversal e com abordagem descritiva.

\section{População}

Participaram do estudo médicos, nutricionistas e enfermeiros que atendem diretamente os idosos nas URSIs. O estudo foi realizado nas sete Unidades da Secretaria Municipal de Saúde de São Paulo. Elas estão distribuídas nos territórios das Coordenadorias Regionais de Saúde (CRS) e dos Distritos Administrativos (DA), conforme segue: Centro-Oeste (1 na Sé), Norte (1 no Tremembé e 1 na Vila Guilherme), Sudeste (1 na Mooca e 1 no Ipiranga), Sul (1 na Cidade Ademar e $1 \mathrm{em}$ Santo Amaro). Esses serviços foram selecionados por se tratarem de unidades especializadas no atendimento da população idosa pertencente à sua área de abrangência.

O grupo investigado foi constituído mediante os seguintes critérios: pertencer às categorias enfermeiro, médico ou nutricionista, e atender diretamente a população, independentemente da especialidade.

No início, a pesquisa previa a entrevista com todos os profissionais de saúde que atuavam nas URSIs. Porém, o grande número de entrevistas e a limitação de tempo levaram à escolha, dentre as categorias profissionais, por enfermeiros, médicos e nutricionistas.

\section{Coleta dos dados}

A coleta de dados aconteceu no período de setembro a novembro de 2015. Os participantes da pesquisa responderam a uma entrevista semiestruturada, cujo roteiro continha dados relacionados ao perfil pessoal e profissional (idade, sexo, profissão, cargo, tempo de ocupação e local de trabalho) e quatro situações referência em relação às necessidades em saúde dos idosos e ao papel do cuidado e da promoção do Envelhecimento Ativo. 
Essas situações foram elaboradas com base em informações obtidas por um profissional de saúde de uma URSI e pelo responsável da Área Técnica de Saúde da Pessoa Idosa da Coordenadoria da Atenção Básica. A experiência desses profissionais possibilitou a aproximação com a realidade dos serviços e permitiu a reflexão sobre o conceito de promoção da saúde na sua prática diária.

Para este artigo, foi selecionada a situação referência apresentada abaixo, tendo em vista que ela abordava as dimensões das necessidades e do cuidado do idoso na rede de atenção à saúde, das contradições do processo de trabalho e da vulnerabilidade no processo de envelhecimento na sociedade. $\mathrm{O}$ desenvolvimento da situação teve base no documento "Envelhecimento ativo: uma política de saúde", na perspectiva da diretriz 1.3 , no que diz respeito à prevenção da iatrogênese no processo de cuidado.

"Sr. Isac, 87 anos de idade, apresenta quadro de hipertensão arterial, diabetes, dislipidemia, dores generalizadas, déficit auditivo e instabilidade postural. Vai à consulta geriátrica desacompanhado, onde relata queixa de constipação intestinal. Para cuidar das comorbidades relatadas acima, o Sr. Isac, faz uso de seis medicamentos. O médico, como de rotina, solicitou exames laboratoriais e prescreveu mais três medicamentos (laxativo, analgésico e relaxante muscular), devido à queixa do paciente. Como paciente era independente e os agravos estavam compensados, o médico não viu necessidade de manter este paciente na URSI e o encaminhou para Unidade Básica de Saúde(UBS) de origem. Se você fosse o médico, manteria esta conduta ou não? Por quê?"

Para a coleta dos dados, usou-se um gravador portátil na aplicação das entrevistas e no registro destas para transcrições no programa QualiQuantiSoft ${ }^{\circledR}$, versão $1.3 \mathrm{c}$.

\section{ANÁLISE E TRATAMENTO DOS DADOS}

O método adotado para o processamento do conteúdo discursivo foi o Discurso do Sujeito Coletivo (DSC), fundamentado na identificação de representações sociais dos profissionais de saúde. Esta técnica consiste em selecionar, de cada resposta individual, as Expressões Chave (ECH), que são trechos mais significativos da entrevista. Às ECH, correspondem Ideias Centrais (IC), que são a síntese do conteúdo discursivo manifestado nas ECH. Cada conjunto de ECH de mesmo sentido ou sentido complementar irá compor, posteriormente, o DSC. Consequentemente, o DSC é um discurso que abrange as $\mathrm{ECH}$ que têm a mesma categoria de $\mathrm{IC}^{(6-7)}$.

Os discursos individuais coletados foram metodologicamente tratados pelo software QualiQuantiSoft ${ }^{\circledR}$, uma vez que, na forma de um ambiente informatizado, o programa organiza dados de natureza qualitativa, como pesquisas de opinião e de representação social, que estejam organizados sob a forma de discurso. Nessa ferramenta, a dimensão qualitativa se expressa no DSC e a quantitativa, na frequência de discursos com mesma ou semelhante IC. Portanto, depois de transcritas e digitadas todas as entrevistas, o programa facilita a classificação em categorias de IC e a elaboração dos discursos, assim como a produção dos relatórios quantitativos, o que fornece o percentual de indivíduos que contribuíram com suas $\mathrm{ECH}$ relativas às $\mathrm{IC}^{(8)}$.
Ressalta-se que um cuidado especial é necessário por parte do pesquisador para selecionar os trechos da entrevista mais relevantes para o tema, assim como para efetuar os recortes e corrigir os desvios e as inadequações desses discursos. Entretanto, é imprescindível que o pesquisador mantenha o fiel sentido do discurso, sem introduzir sua interpretação ou opinião. Assim, o software poderá executar as classificações tendo como base elementos factuais oriundos diretamente das entrevistas dos sujeitos ${ }^{(8)}$.

Por fim, os DSC produzidos foram analisados de acordo com a articulação em relação aos princípios das Nações Unidas para idosos no que diz respeito ao pilar saúde, contido no projeto "Envelhecimento ativo: uma política de saúde"(1).

\section{Aspectos ÉTICOS}

O estudo foi avaliado e aprovado pelo Comitê de Ética em Pesquisa da Universidade São Judas Tadeu, sob o Parecer no 1.220.070/2015 e pela Secretaria Municipal de Saúde de São Paulo, sob o Parecer no 1.221.399/2015. Respeitaram-se as normas e as diretrizes para a realização de pesquisas que envolvem seres humanos, em observância às determinações presentes na resolução 466/12, do Conselho Nacional de Saúde. Na execução, asseguraram-se o sigilo e o anonimato dos profissionais, assim como a privacidade e a liberdade de participar ou não da pesquisa e de retirar-se desta a qualquer momento, com o uso do Termo de Consentimento Livre e Esclarecido. Os entrevistados foram identificados com o termo genérico "Ursi" e números subsequentes, conforme a ordem em que a coleta de dados foi realizada, por exemplo: Ursi01, Ursi02, e assim por diante.

\section{RESULTADOS}

Dos 31 profissionais contatados que corresponderam aos critérios definidos, dois recusaram-se a participar.

De acordo com a Tabela 1, entre os 29 que participaram, $16(55,2 \%)$ eram médicos, $7(24,1 \%)$ nutricionistas e 6 $(20,7 \%)$ enfermeiros. Essas porcentagens refletem a distribuição dos profissionais da amostra selecionada, com maior proporção do sexo feminino (79,3\%), na faixa etária de 40 anos ou mais $(62,1 \%)$. Em relação ao tempo de trabalho nas URSIs, observou-se um predomínio de 1 a 5 anos.

Tabela 1 - Caracterização dos profissionais de saúde entrevistados nas URSIs, segundo sexo, faixa etária, profissão e tempo de trabalho - São Paulo, SP, Brasil, 2015.

\begin{tabular}{lcc}
\hline Variável & N & $\%$ \\
\hline Total de profissionais & 29 & 100,0 \\
Sexo & & \\
Feminino & 23 & 79,3 \\
Masculino & 6 & 20,7 \\
Faixa Etária & 11 & \\
$<40$ & 18 & 37,9 \\
$\geq 40$ & & 62,1 \\
\hline
\end{tabular}




\begin{tabular}{lcc}
...continuação & N & $\%$ \\
\hline Variável & & \\
\hline Profissão & 16 & 55,2 \\
Médico & 6 & 20,7 \\
Enfermeiro & 7 & 24,1 \\
Nutricionista & & \\
Tempo de URSI & 8 & 27,6 \\
$<1$ ano & 14 & 48,3 \\
$1-5$ anos & 7 & 24,1 \\
$>5$ anos & &
\end{tabular}

Da situação referência selecionada, emergiram 62 IC, identificadas nas entrevistas com os profissionais de saúde e agrupadas nas seguintes categorias: A - Encaminhamento do paciente para Unidade Básica de Saúde; B - Discordância: questão da polifarmácia; C - Comorbidades apontam necessidades de nutricionista; $\mathrm{D}$ - Discordância da conduta médica: manutenção do paciente na URSI; E - Protocolo de avaliação do paciente; F - Envolvimento da família no tratamento; G - Importância da equipe multidisciplinar. Com base nas categorias de IC consolidadas, foram construídos sete DSC.

A Figura 1 apresenta a distribuição das respostas dos 29 sujeitos da pesquisa, por categoria de IC, identificadas nos discursos. Reiterando-se que o discurso de cada profissional pode ser inserido em mais de uma categoria, verifica-se maior frequência nas categorias D $(82,8 \%$, ou seja, 24 dos 29 profissionais), B (31\%, ou seja, 9 dos 29 profissionais), C e E (24,1\%, ou seja, 7 dos 29 profissionais).

\section{Categorias de Ideias Centrais}

A - Encaminhamento do paciente para Unidade Básica de Saúde

B - Discordância: questão da polifarmácia

C - Comorbidades apontam necessidades de nutricionista

D - Discordância da conduta médica: manutenção do paciente na URSI

E - Protocolo de avaliação do paciente

F - Envolvimento família no tratamento

G - Importância da equipe multidisciplinar

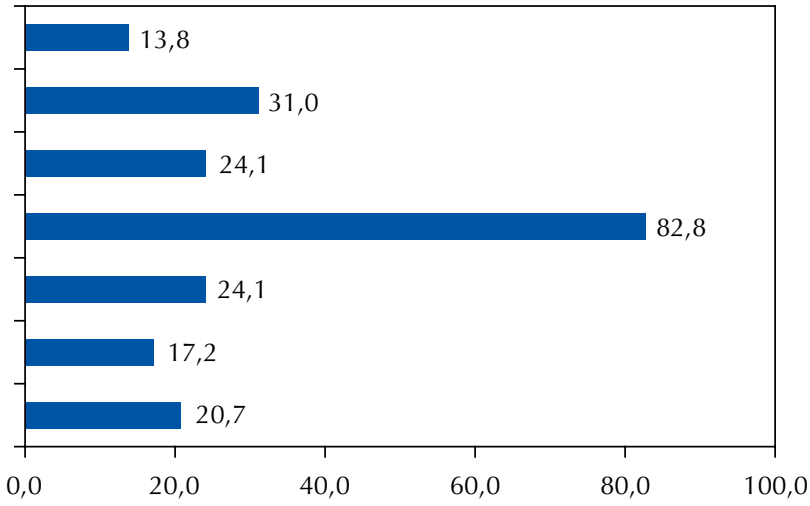

(\%)

Nota: $(\mathrm{n}=29)$

Figura 1 - Distribuição do percentual dos profissionais segundo categorias de Ideias Centrais identificadas nos discursos - São Paulo, SP, Brasil, 2015.

\section{Categoria de Ideia Central A - Encaminhamento do PACIENTE PARA UnIDADE BásICA DE SAúde}

DSC 1: Partindo do pressuposto de que este geriatra avaliou o paciente, que já passou por nutricionista, já passou por otorrino, já passou pela fisioterapia, já passou pelo grupo de quedas, e está estabilizado, ele iria para UBS, porque aqui, na URSI, basicamente, assim, entra, são prioridades os pacientes que têm transtorno cognitivo, pacientes que têm quedas de repetição. Portanto, se o paciente tá compensado em todo seu quadro, ele deve, sim, ser encaminhado para sua Unidade Básica de origem, porque essa é a proposta da URSI (Ursi 01, Ursi03, Ursi 05 e Ursi19).

O DSC mencionado contemplou a diretriz 1.1 na meta prevenção e tratamentos eficazes por meio de serviços de triagem mais humanizados e processos terapêuticos mais eficientes e baratos. Identificaram-se também no DSC algumas das metas preconizadas na diretriz 1.3, que estabelece a necessidade de se desenvolver um contínuo de serviços sociais e de saúde acessíveis, de qualidade e adequados para a terceira idade, especificamente no que diz respeito à continuidade da assistência durante o curso da vida, com base na promoção da saúde e na prevenção de doenças, bem como no direito ao acesso justo e equitativo aos serviços e sistemas de cuidados éticos coordenados.

Resume-se na Figura 2 a correspondência entre situação referência, ECH e IC que emergiram da entrevista semiestruturada, a análise e o tratamento de dados com relação à categoria $\mathrm{A}$, seu respectivo DSC e a relação com o preconizado no Documento de Referência, utilizado para a análise das representações sociais dos profissionais das URSIs. As demais categorias de IC e DSC seguiram a mesma linha de análise. 


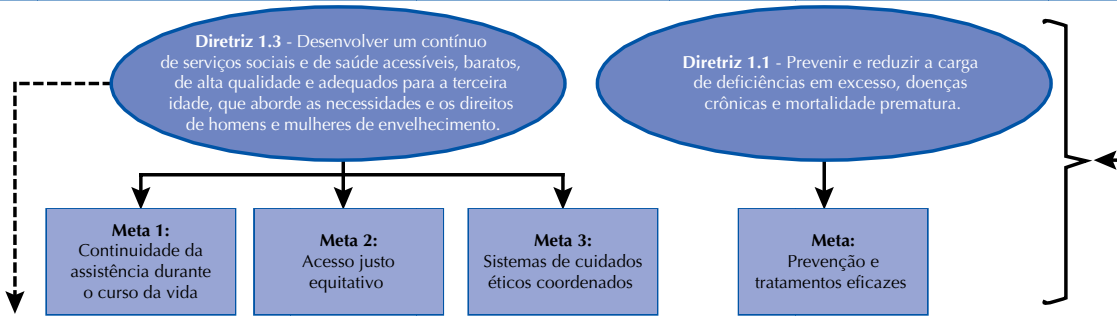

Situação referência: Sr. Isac

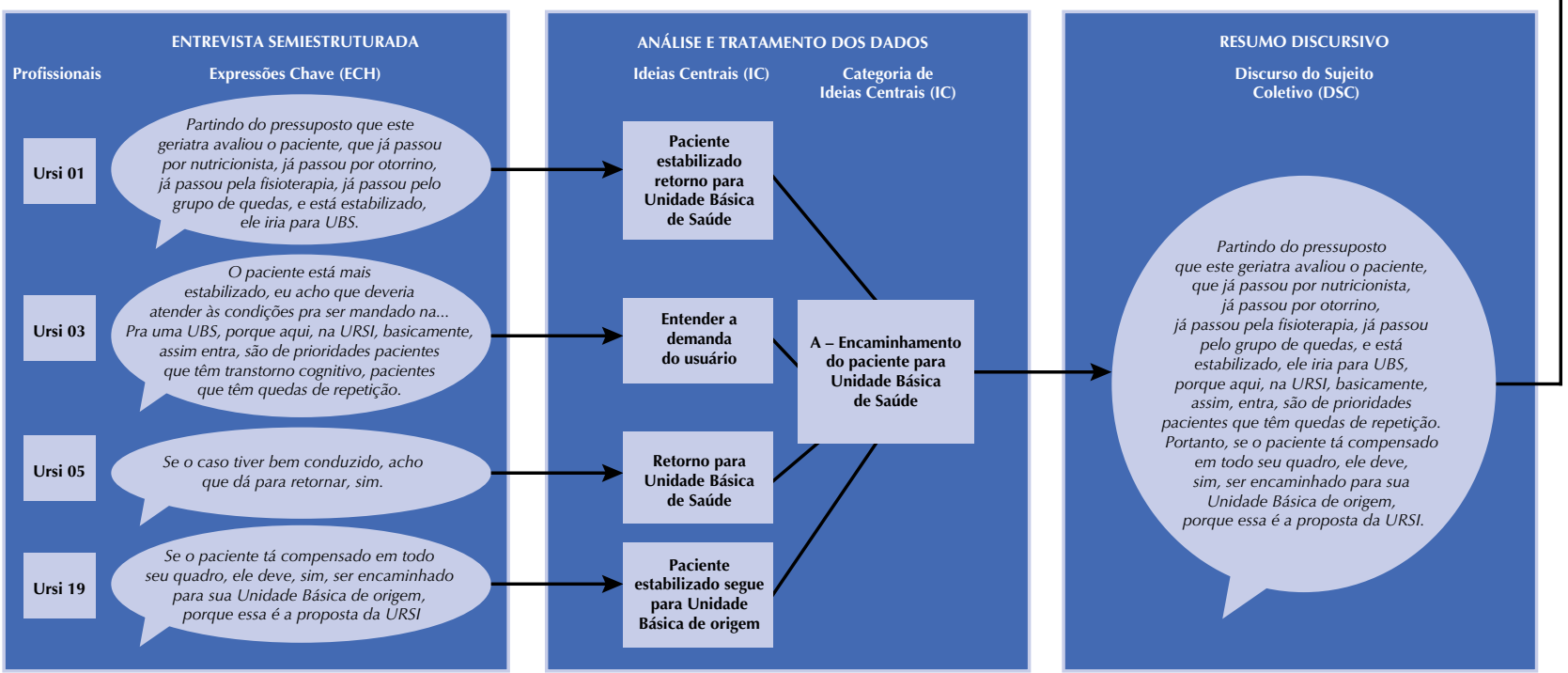

Figura 2 - Representação esquemática da construção do Discurso do Sujeito Coletivo a partir da entrevista semiestruturada e sua relação com as diretrizes e metas do documento "Envelhecimento ativo: uma política de saúde".

\section{Categoria de Ideia Central B - Discordância: QUESTÃO DA POLIFARMÁCIA}

DSC 2: Se fosse um paciente que eu estivesse atendendo, antes de mais nada tentaria diminuir essa medicação. Eu o acompanharia por algumas consultas, tentando diminuir a lista de medicações que ele usa-da polifarmácia. Ele já faz uso de seis medicamentos e o geriatra introduziu mais três, entre eles relaxantes musculares, o que, inclusive, pode aumentar a sonolência, e também o risco de queda. Então, revisar medicamentos que já estão em uso - se são mesmo necessários - e evitar ao máximo agregar mais medicamentos à prescrição dele. Ele vem à consulta sozinho, mas ele merece ter tratamento da URSI justamente para controlar essas variáveis e evitar iatrogenia; evitar que os remédios se somem, e então, assim, prevenir esses eventos, inclusive polifarmácia (Ursi 02, Ursi03, Ursi05, Ursi11, Ursi15, Ursi17, Ursi22, Ursi23 e Ursi24).

No DSC acima, observaram-se conteúdos: da diretriz 1.2, que prevê a redução dos fatores de risco associados às principais doenças e o aumento dos fatores de proteção, no que se refere à meta de reduzir a prescrição inadequada de medicamentos por profissionais da saúde; e da diretriz 1.3, que originou a situação referência na meta de prevenção de iatrogênese.

\section{Categoria de Ideia Central C - Comorbidades APONTAM NECESSIDADE DE NUTRICIONISTA}

DSC 3: Eu encaminharia o seu Isac para uma nutricionista, a fim de fazer reeducação alimentar, uma vez que ele tem hipertensão arterial, diabetes e dislipidemia. É importante fazer uma avaliação nutricional, para ter uma orientação dietética melhor, tá? Basicamente, aumentar as fibras e ver o quanto de líquido ele toma. Orientar uma alimentação mais laxante, natural, né? Com fibras, tudo o mais, eu não utilizaria laxante(...). (Ursi03, Ursi05, Ursi06, Ursi12, Ursi13, Ursi14 e Ursi18).

Esse DSC, além de contemplar a diretriz 1.1, que propõe prevenir e reduzir a carga de deficiências em excesso, doenças crônicas e mortalidade precoce, especificamente na meta prevenção e tratamentos eficazes, abordou a diretriz 1.2 nas metas nutrição e alimentação saudável, com o objetivo de assegurar uma alimentação adequada à faixa etária durante toda a vida. 


\section{Categoria de Ideia Central D - Discordância da} CONDUTA MÉDICA: MANUTENÇ̃̃O DO PACIENTE NA URSI

DSC 4: É um paciente que tem total indicação para ser mantido por um periodo, pelo menos, na URSI, até que você possa resolver melhor todas essas questöes. Mesmo ele tendo uma independência, os agravos são fatos suficientes pra que ele seja mantido na URSI. Muitas medicações, instabilidade postural, déficit auditivo, desacompanhado. Ele é hipertenso, diabético, dislipidêmico, tem dor generalizada e constipação intestinal. Então é um paciente que tem multimorbidades, né? E não só pelas multimorbidades, mas também pela polifarmácia, é um paciente que tem realmente indicação de manter o acompanhamento na URSI. Então eu não mandaria para UBS de jeito nenhum! (Ursi02, Ursi04, Ursi06, Ursi07, Ursi08, Ursi09, Ursi10, Ursi11, Ursi13, Ursi14, Ursi15, Ursi16, Ursi17, Ursi18, Ursi20, Ursi21, Ursi22, Ursi23, Ursi24, Ursi25, Ursi26, Ursi27, Ursi28 e Ursi29).

O DSC 4 referendou a diretriz 1.1 na meta prevenção e tratamentos eficazes, bem como a diretriz 1.3 nas metas continuidade da assistência durante o curso da vida e acesso justo e equitativo, ambas diretrizes também relacionadas ao DSC 1.

\section{Categoria de Ideia Central E - Protocolo de AVALIAÇÃO DO PACIENTE}

DSC 5: Aqui a gente está trabalhando com um material, um modelo, que foi desenvolvido agora, principalmente para esses pacientes mais idosos, com instabilidade. É aplicado um questionário, que se chama AMPI, e vê o grau de idoso que ele éidoso saudável, pré-frágil ou idoso frágil. Se realmente constar que esse paciente, mesmo com doença crônica, tem um grau de independência, ele acaba voltando para a Unidade de origem, ele não tem necessidade de estar numa URSI e, à medida que for solicitada uma nova avaliação geriátrica, ele volta pro geriatra, então, não é que ele vai embora e depois não volta mais (Ursi01, Ursi03, Ursi05, Ursi08, Ursi09, Ursi22 e Ursi27).

Observou-se nesse DSC conteúdo das diretrizes 1.1 na meta prevenção e tratamentos eficazes e 1.3 nas metas continuidade da assistência durante o curso da vida, acesso justo e equitativo aos serviços de atenção em saúde e sistemas de cuidados éticos e coordenados.

\section{Categoria de Ideia Central F - Envolvimento FAMILIAR NO TRATAMENTO}

DSC 6: Primeiro de tudo, eu pediria que ele viesse com um acompanhante nas consultas, pra diminuir o risco desse paciente nas ruas, desacompanhado. As vezes orientar um plano de cuidado, assim, tudo, pro familiar, ou para alguém, para aí justificar o porquê de ele estar indo pra UBS. Porque, geralmente, a pessoa idosa, ela acha que é um peso na vida do filho, e ela não quer que o filho venha, saia do serviço pra vir acompanhar. Mas no caso, a família teria que acompanhar (Ursi08, Ursi18, Ursi21, Ursi24 e Ursi26).

Nesse DSC, evidenciaram-se as diretrizes: 1.1 na meta melhorar a qualidade de vida de pessoas com doenças crônicas e deficiências, favorecer sua independência contínua e interdependência e apoio comunitário para os familiares; e 1.4, que preconiza treinamento e educação para cuidadores, na meta cuidadores informais.

\section{Categoria de Ideia Central G - ImportânCia dA EQUIPE MULTIDISCIPLINAR}

DSC 7: Ficando na URSI, ele teria acesso ao atendimento multidisciplinar, né? (...) Então, poderia ser encaminhado pra nutricionista, psicólogo; precisa de assistente social, precisa de terapeuta ocupacional, poderia ser encaminhado pro fisioterapeuta, para ajudar nessas dores, né, generalizadas. O déficit auditivo, poderia encaminhar pra fono, né? Até porque a gente sabe que a URSI, como modelo de São Paulo, tem uma equipe multidisciplinar, e as UBSs não (Ursi07, Ursi13, Ursi16, Ursi18, Ursi26 e Ursi28).

No DSC 7, destacam-se conteúdos da diretriz 1.2, que prevê a redução dos fatores de risco associados às principais doenças e o aumento dos fatores de proteção, como incentivo à prática de atividade física, garantia de alimentação saudável e desenvolvimento da autonomia e das habilidades cognitivas, melhorando o bem-estar psicológico; assim como os da diretriz 1.3, na meta continuidade da assistência durante o curso da vida, baseando-se na promoção da saúde e na prevenção de doenças.

À medida que as categorias de IC iam se delineando e os DSC sendo produzidos, foram analisados de acordo e em articulação com as diretrizes e metas do documento de referência da pesquisa (Quadro 1).

Quadro 1 - Categorias de Ideias Centrais, Discurso do Sujeito Coletivo e suas relações com as diretrizes e metas do documento de referência - São Paulo, SP, Brasil, 2015.

\begin{tabular}{|l|c|c|c|}
\hline Categoria de Ideia Central (IC) & $\begin{array}{c}\text { Discurso do Sujeito } \\
\text { Coletivo (DSC) }\end{array}$ & Diretriz & Meta \\
\hline $\begin{array}{l}\text { A - Encaminhamento do paciente para } \\
\text { Unidade Básica de Saúde }\end{array}$ & DSC 1 & 1.1 & Prevenção e tratamentos eficazes \\
\hline B - Discordância: questão da polifarmácia & DSC 2 & 1.3 & $\begin{array}{c}\text { Continuidade da assistência durante o curso da vida } \\
\text { Acesso justo e equitativo } \\
\text { Sistemas de cuidados éticos coordenados }\end{array}$ \\
\hline & & 1.3 & Medicamentos \\
\hline
\end{tabular}


...continuação

\begin{tabular}{|c|c|c|c|}
\hline Categoria de Ideia Central (IC) & $\begin{array}{l}\text { Discurso do Sujeito } \\
\text { Coletivo (DSC) }\end{array}$ & Diretriz & Meta \\
\hline \multirow[t]{2}{*}{$\begin{array}{l}\text { C - Comorbidades apontam necessidades de } \\
\text { nutricionista }\end{array}$} & DSC 3 & 1.1 & Prevenção e tratamentos eficazes \\
\hline & & 1.2 & $\begin{array}{l}\text { Nutrição } \\
\text { Alimentação saudável }\end{array}$ \\
\hline \multirow[t]{2}{*}{$\begin{array}{l}\text { D - Discordância da conduta médica: } \\
\text { manutenção do paciente na URSI }\end{array}$} & DSC 4 & 1.1 & Prevenção e tratamentos eficazes \\
\hline & & 1.3 & $\begin{array}{l}\text { Continuidade da assistência durante o curso da vida } \\
\text { Acesso justo e equitativo }\end{array}$ \\
\hline \multirow[t]{2}{*}{ E - Protocolo de avaliação do paciente } & DSC 5 & 1.1 & Prevenção e tratamentos eficazes \\
\hline & & 1.3 & $\begin{array}{c}\text { Continuidade da assistência durante o curso da vida } \\
\text { Acesso justo e equitativo } \\
\text { Sistemas de cuidados éticos coordenados }\end{array}$ \\
\hline \multirow[t]{2}{*}{ F - Envolvimento familiar no tratamento } & DSC 6 & 1.1 & Qualidade de vida \\
\hline & & 1.4 & Cuidadores informais \\
\hline \multirow[t]{2}{*}{ G - Importância da equipe multidisciplinar } & DSC 7 & 1.2 & $\begin{array}{l}\text { Atividade física } \\
\text { Nutriçãa } \\
\text { Alimentação Saudável } \\
\text { Fatores psicológicos }\end{array}$ \\
\hline & & 1.3 & Continuidade da assistência durante o curso da vida \\
\hline
\end{tabular}

\section{DISCUSSÃO}

Os idosos não constituem um grupo homogêneo e a diversidade entre os indivíduos costuma aumentar com a idade $^{(1)}$. Tal população, entretanto, apresenta particularidades já bem determinadas: maior prevalência de doenças crônicas, que geram mais custos; maiores fragilidades e menor disponibilidade de recursos sociais e financeiros, tanto no nível individual quanto da sociedade como um todo ${ }^{(9)}$.

Em função dessas características, a maioria dos modelos assistenciais vigentes, em se tratando do cuidado ao idoso, foca exclusivamente a doença, com multiplicação de consultas de especialistas, informação não compartilhada, prescrição de inúmeros fármacos potencialmente inapropriados e risco de interação inadequada de várias drogas. Consequentemente, os casos de iatrogenia nesta população são altamente prevalentes ${ }^{(9-10)}$.

Estudos recentes questionam este modelo, propondo "que a atenção deva ser organizada de maneira integrada, e os cuidados, coordenados ao longo do percurso assistencial numa lógica de rede" ${ }^{\text {(11). }}$.

As representações sociais dos sujeitos desta pesquisa, articulados nos DSC, explicitam todas essas contradições vivenciadas no cotidiano das URSIs.

Observa-se falta de consenso entre os profissionais de saúde quanto aos modelos de atenção revelados no DSC 1 e no DSC 4. De todos os entrevistados, 24 manteriam este paciente na URSI ('É um paciente que tem total indicação para ser mantido por um periodo, pelo menos, na URSI...'), e 4 o encaminhariam para a UBS de origem ('... Portanto, se o paciente tá compensado em todo seu quadro, ele deve, sim, ser encaminhado para sua Unidade Básica de origem...').

Estudos baseados em "evidências demonstram que o modelo de atenção à saúde do idoso, para ser eficiente, deve compreender atividades organizadas num fluxo hierarquizado, e que, mesmo sendo executadas de forma independente, se inter-relacionem" ${ }^{12)}$. De fato, a Atenção Básica, como proposta na Política Nacional de Atenção Básica, constitui o contato inicial dos usuários na rede de saúde e "caracteriza-se por desenvolver um conjunto de ações, nos âmbitos individual e coletivo, que abrangem a promoção e a proteção à saúde, a prevenção de agravos, o diagnóstico, o tratamento, a reabilitação e a manutenção da saúde"(13).

Para garantir a longitudinalidade do cuidado, ganha destaque a estratégia das Redes de Atenção à Saúde (RAS). Ela objetiva a integração de ações e serviços de saúde pautados na atenção contínua, integral e humanizada, além de possibilitar o melhor desempenho do SUS em relação ao acesso, à equidade, à eficácia clínica e sanitária e à eficiência econômica ${ }^{(14)}$.

É fundamental reconhecer que a integralidade da atenção à saúde da população não se resume a um único serviço ou sistema de saúde. A dimensão da integralidade se estabelece pela composição de uma rede solidária e intersetorial de políticas, serviços e profissionais ${ }^{(15)}$.

Encaminhar o paciente para a UBS de origem ou mantê-lo na própria URSI gerou dúvidas na conduta prática dos profissionais de saúde. Talvez esse impasse evidencie que, embora reconhecendo a importância de um trabalho multidisciplinar conforme o DSC 7 ('Ficando na URSI, ele teria acesso ao atendimento multidisciplinar...'), essa estratégia ainda está pouco fortalecida e distante do interdisciplinar. Ao lado disso, no DSC 5, observa-se que os profissionais não se apropriaram totalmente do entendimento e da validade dos protocolos que regem o processo de trabalho, o fluxo de encaminhamentos e as atribuições de cada nível da assistência: ('Aqui agente está trabalhando com um material, um modelo, que foi desenvolvido agora (...) um questionário, que se chama AMPI...'). Os Protocolos Clínicos, as Diretrizes Terapêuticas 
e os Cadernos de Atenção Básica são importantes ferramentas de apoio a essa gestão do cuidado, merecendo atenção especial e conhecimento pelos profissionais. $\mathrm{O}$ uso desses instrumentos tende a minimizar a variabilidade das informações e condutas entre os membros da equipe de saúde, estabelecendo limites de ação e cooperação entre os diversos profissionais ${ }^{(16)}$. Estudo realizado com profissionais de UTI, relativo aos cuidados paliativos, também evidenciou visões diferentes da eficácia do cuidado na recuperação do paciente, a dificuldade na colaboração interdisciplinar e ausência de protocolos assistenciais, o que dificulta a tomada de decisão dos cuidados ${ }^{(17)}$. No caso do Sr. Isac, fica clara a importância de um processo de treinamento e educação permanente para a utilização dos protocolos preestabelecidos para a assistência oferecida na URSI. Entender a demanda de cada caso se torna essencial para uma conduta adequada da equipe multidisciplinar da URSI.

A questão da polifarmácia emerge como um outro aspecto do modelo de atenção, centrado na doença, que revela a preocupação dos profissionais - conforme o DSC 2 ('Se fosse um paciente que eu estivesse atendendo, antes de mais nada tentaria diminuir essa medicação. Eu o acompanharia por algumas consultas, tentando diminuir a lista de medicaçôes que ele usa - da polifarmácia...'). A polifarmácia associa-se a um número importante de iatrogenia e desencadeamento de efeitos colaterais e reações adversas em idosos, comprometendo sua funcionalidade ${ }^{(18-19)}$. Estudo qualitativo realizado com usuários cadastrados nas Unidades de Saúde da Família na Bahia mostra o mesmo questionamento por parte dos trabalhadores quanto ao caráter prescritivo-curativo das consultas médicas, que nem sempre propicia a escuta e o conhecimento das necessidades em saúde, enfatizando os aspectos biomédicos ao valorizar a prescrição de medicamentos ${ }^{(20)}$.

Aliado a esse DSC, e levando em consideração a queixa do paciente, os profissionais de saúde identificaram a importância da intervenção nutricional como fator positivo no tratamento, evitando, assim, a introdução de mais medicamentos e suas consequências na saúde do idoso, de acordo com o exposto no DSC 3 ('...E É importante fazer uma avaliação nutricional, para ter uma orientação dietética melhor, tá? Basicamente, aumentar as fibras e ver o quanto de líquido ele toma. Orientar uma alimentação mais laxante, natural, né? Com fibras, tudo o mais, eu não utilizaria laxante...'). Concorda-se que a nutrição tem papel importante no processo do envelhecimento, na etiologia de doenças associadas com a idade, bem como no declínio funcional e no surgimento de deficiências; assim, a avaliação e o monitoramento nutricional de idosos são necessários para uma assistência adequada e para o planejamento de ações de promoção da saúde ${ }^{(21)}$. Outro aspecto suscitado pelo DSC 3 foi a importância da ingestão de líquidos, cujo descuido ou esquecimento pelo idoso representa um risco de desidratação. Estes podem advir de várias causas, como a diabetes, demência e o próprio fator envelhecimento, que diminui os mecanismos de regulação da sede ${ }^{(22-23)}$.

O DSC 6 ressalta a importância do envolvimento familiar no tratamento do idoso ('Primeiro de tudo, pediria que ele viesse com um acompanhante nas consultas, pra diminuir o risco desse paciente nas ruas, desacompanhado. As vezes orientar um plano de cuidado, assim, tudo, pro familiar, ou para alguém, para aí justificar o porquê de ele estar indo pra UBS...'). As políticas de saúde relacionadas aos idosos preconizam que as pessoas mais velhas e seus cuidadores precisam estar envolvidos no planejamento e na implementação de atividades para ampliar o saber relacionado ao envelhecimento ativo e seu cuidado ${ }^{(1)}$, corroborando o DSC. A doença crônica nessa situação não é sinônimo de inutilidade ou de dependência, e a família exerce papel fundamental nestas circunstâncias, pois pode reforçar nos idosos o cuidado consigo mesmos, propiciando o exercício da sua autonomia ${ }^{(24)}$.

No referido discurso, destaca-se ainda a resistência do paciente que não quer atrapalhar a rotina do familiar ('...A pessoa idosa, ela acha que é um peso na vida do filho, e ela não quer que o filho venha, saia do serviço pra vir acompanhar'). Atualmente, os idosos estão muito mais propensos a envelhecer bem do que se tornarem dependentes. A velhice não está mais associada à doença nem à perda do domínio sobre a própria vida. Em particular, o idoso quer que sua vontade seja considerada, assim como poder tomar as decisões que afetam sua autonomia e sua qualidade de vida, inclusive em caso de doenças crônicas ${ }^{(25)}$.

Tentar resgatar vínculos familiares desse idoso, entender o meio em que vive e quem é sua rede de apoio, são fatores que podem contribuir para o cuidado com o acompanhamento do caso e para a aderência ao tratamento.

A adoção do modelo com foco na promoção da saúde e do fortalecimento da RAS incorporaria "pelo menos dois elementos fundamentais para o cuidado do idoso: a diminuição de iatrogenia e a organização do fluxo de cuidados. As diretrizes e os protocolos clínicos também são essenciais na construção do projeto terapêutico"(11), "fio condutor para o fluxo da linha do cuidado, que estabelece o percurso assistencial de acordo com as necessidades"(12).

Aponta-se como limitação do presente estudo o fato de não terem sido entrevistados todos os profissionais que compunham as equipes de saúde das URSIs, os quais poderiam ter trazido outros enfoques em relação às necessidades e ao cuidado dos idosos na busca do envelhecimento ativo. Este estudo limita-se também pela não inclusão nos DSC da articulação com outros setores, como assistência social, pessoas com deficiência e mesmo uma compreensão das questões socioeconômicas e culturais que envolvem esse idoso e sua família no território em que vivem. Em contrapartida, aponta-se como potência o fato de a pesquisa ter sido desenvolvida na totalidade das URSIs do município de São Paulo.

Vislumbra-se a possibilidade de continuidade do estudo, incluindo todos os profissionais de saúde envolvidos no processo, além dos gestores dos serviços e demais atores dessa rede, como representantes de usuários de conselhos gestores das unidades. Conhecer as percepções dos idosos atendidos nas URSIs e dos seus familiares também seria uma abordagem importante para novas pesquisas, o que permitiria incorporar as dimensões biopsicossociais e espirituais do envelhecimento. 
Em razão dos achados deste estudo, sugere-se a elaboração de planos de educação permanente para os profissionais das URSIs que envolvam desde os protocolos clínicos e fluxos de referência e contrarreferência, como reflexão e revisão das práticas de cuidado com base na Política Nacional de Promoção da Saúde, nos Cadernos de Atenção Básica - Envelhecimento e Saúde da Pessoa Idosa, nas Redes de Atenção à Saúde, entre outros.

\section{CONCLUSÃO}

Ao retomar o objetivo central do estudo, verificou-se que os profissionais conseguem pautar suas ações nas necessidades de saúde dos idosos, uma vez que, na análise dos DSC, identificaram-se conteúdos que abordaram as quatro diretrizes do documento do envelhecimento ativo.

Com relação ao seu papel no cuidado e na promoção do Envelhecimento Ativo, os DSC apontam na direção da construção de um cuidado multidisciplinar, com práticas que tenham como base a clínica ampliada.

Desta forma, esta pesquisa estimula a reflexão da rede de atenção quanto a medidas a serem tomadas para melhoria da qualidade no cuidado e na promoção do Envelhecimento Ativo para a população que foi objeto do estudo.

\section{RESUMO}

Objetivo: Identificar as representações sociais de profissionais de saúde que atendem idosos nas sete Unidades de Referência à Saúde do Idoso no município de São Paulo quanto às necessidades em saúde, seu papel no cuidado e na promoção do envelhecimento ativo. Método: Pesquisa qualitativa, fundamentada nas diretrizes do documento "Envelhecimento ativo: uma política de saúde" da Organização Mundial da Saúde. Utilizou-se o Discurso do Sujeito Coletivo para sistematizar as representações sociais, cujas ideias centrais foram categorizadas utilizando-se o software QualiQuantiSoft ${ }^{\circledR}$. Foram elaboradas quatro situações referência e selecionou-se aquela relacionada à questão da iatrogênese e vulnerabilidade do idoso. Resultados: Participaram do estudo 29 profissionais (16 médicos, 7 nutricionistas e 6 enfermeiros). Dos discursos dos entrevistados, emergiram sete categorias de ideias centrais, com maior frequência para discordância da conduta médica quanto ao encaminhamento do paciente à Unidade Básica de Saúde e o problema da polifarmácia. Conclusão: Os discursos dos profissionais apontam a necessidade da construção de um cuidado que supere a fragmentação do processo de trabalho.

\section{DESCRITORES}

Envelhecimento; Idoso; Políticas Públicas; Profissional de Saúde; Serviços de Saúde para Idosos.

\section{RESUMEN}

Objetivo: Identificar las representaciones sociales de los profesionales de la salud que atienden a los ancianos en las siete Unidades de Referencia para la Salud de los Ancianos en la ciudad de São Paulo con respecto a las necesidades de salud, su papel en la atención y promoción del envejecimiento activo. Método: Estudio cualitativo, basado en las directrices del documento "Envejecimiento activo: un marco político" de la Organización Mundial de la Salud. El Discurso del Sujeto Colectivo se utilizó para sistematizar las representaciones sociales, cuyas ideas centrales se clasificaron utilizando el software QualiQuantiSoft ${ }^{\circledR}$. Se elaboraron cuatro situaciones de referencia y se seleccionó la relacionada con el tema de iatrogénesis y vulnerabilidad de los ancianos. Resultados: Veintinueve profesionales (16 médicos, 7 nutricionistas y 6 enfermeros) participaron en el estudio. De los discursos de los entrevistados, surgieron siete categorías de ideas centrales, con mayor frecuencia para el desacuerdo de la conducta médica con respecto a la derivación del paciente a la Unidad Básica de Salud y el problema de la polifarmacia. Conclusión: Los discursos de los profesionales apuntan a la necesidad de crear una atención que supere la fragmentación del proceso de trabajo.

\section{DESCRIPTORES}

Envejecimiento; Anciano; Políticas Públicas; Personal de Salud; Servicios de Salud para Ancianos.

\section{REFERÊNCIAS}

1. Organização Mundial de Saúde. Envelhecimento ativo: uma política de saúde [Internet]. Brasília: OPAS; 2005 [citado 2018 jun. 10 ]. Disponível em: http://bvsms.saude.gov.br/bvs/publicacoes/envelhecimento_ativo.pdf

2. Kalache A. Vested rights, active aging and resiliance: the importance of such concepts throughout life [editorial]. Rev Bras Geriatr Gerontol [Internet]. 2017 [cited 2018 Nov 02];20(2):159-60. Available from: http://www.scielo.br/scielo.php?script=sci_arttext\&pid=S180998232017000200159\&lng=pt

3. Carnut L. Cuidado, integralidade e atenção primária: articulação essencial para refletir sobre o setor saúde no Brasil. Saúde Debate [Internet]. 2017 [citado 2018 nov. 3];41(115):1177-86. Disponível em: http://www.scielo.br/scielo.php?script=sci_arttext\&pid=S0103$11042017000401177 \& \operatorname{lng}=$ pt\&tlng=pt

4. São Paulo (Cidade). Secretaria Municipal de Saúde; Coordenação de Atenção Básica. Documento norteador: Unidade de Referência à Saúde do Idoso URSI [Internet]. São Paulo: PMSP; 2016 [citado 2018 nov. 2]. Disponível em: https://www.prefeitura.sp.gov.br/cidade/ secretarias/upload/saude/DOCUMENTONORTEADOR\%20URSIversaofinal09012017.pdf

5. Marková I. The making of the theory of social representations. Cad Pesq [Internet]. 2017 [cited 2018 Nov 2];47(163):358-75. Available from: http://www.scielo.br/scielo.php?pid=S0100-15742017000100358\&script=sci_arttext\&tlng=en

6. Lefèvre F, Lefèvre AMC. Pesquisa de representação social: um enfoque qualiquantitativo: a metodologia do discurso do sujeito coletivo. Brasília: Líber Livro; 2010.

7. Morais VS, Chaves APL. Perception of municipal health managers regarding environmental health: Cerrado Araguaia Tocantins intermunicipal health consortium. Saude Soc. [Internet] 2016 [cited 2018 Nov 2];25(2):349-60. Available from: http://www.scielo.br/ scielo.php?script=sci_arttext\&pid=S0104-12902016000200349\&lng=en\&nrm=iso\&tlng=en

8. Nicolau KW, Escalda PMF, Furlan PG. Método do Discurso do Sujeito Coletivo e usabilidade dos softwares Qualiquantisoft e DSCsoft na pesquisa qualiquantitativa em saúde. Fronteiras J Soc Technol Envirom Sci [Internet]. 2015[citado 2018 dez. 5];4(3). Disponível em: http://periodicos.unievangelica.edu.br/index.php/fronteiras/article/view/1413 
9. Veras RP, Oliveira M. Aging in Brazil: the building of a healthcare model. Ciênc Saúde Coletiva [Internet]. 2018 [cited 2018 Dec 20];23(6):1929-36. Available from: http://www.scielo.br/scielo.php?pid=S1413-81232018000601929\&script=sci_arttext\&tlng=en

10. Novaes PH, Cruz DT, Lucchetti ALG, Leite ICG, Lucchetti, G. The "iatrogenic triad": polypharmacy, drug-drug interactions, and potentially inappropriate medications in older adults. Int J Clin Pharm. 2017;39(4):818-25. DOI: http://dx.doi.org/10.1007/s11096-017-0470-2

11. Veras R. Care pathway for the elderly: detailing the model. Rev Bras Geriatr Gerontol [Internet]. 2016 [cited 2019 May 20];19(6):887-905. Available from: http://www.scielo.br/pdf/rbgg/v19n6/1809-9823-rbgg-19-06-00887.pdf

12. Oliveira MR, Veras RP, Cordeiro HA, Pasinato MT. A mudança de modelo assistencial de cuidado ao idoso na Saúde Suplementar: identificação de seus pontos-chave e obstáculos para implementação. Physis [Internet]. 2016 [citado 2019 May 22];26(4):1383-94. Disponível em: http://www.scielo.br/pdf/physis/v26n4/1809-4481-physis-26-04-01383.pdf

13. Bara VMF, Paz EPA, Guimarães RM, Silva BF, Gama BBM, Moratelli L. Diagnóstico de utilização do instrumento de avaliação da atenção primária à saúde - PCATool-Brasil versão adulto - para população idosa. Cad Saúde Colet [Internet]. 2015 [citado 2019 nov. 5]; 23(3):330-5. Disponível em: http://www.scielo.br/scielo.php?script=sci_arttext\&pid=S1414-462X2015000300330\&lng=pt

14. Brasil. Ministério da Saúde. Portaria n. 2.436, de 21 de setembro de 2017. Aprova a Política Nacional de Atenção Básica, estabelecendo a revisão de diretrizes para a organização da Atenção Básica, no âmbito do Sistema Único de Saúde (SUS) [Internet]. Brasília; 2017 [citado 2018 nov. 4]. Disponível em: http://bvsms.saude.gov.br/bvs/saudelegis/gm/2017/ prt2436_22_09_2017.html

15. Silva PFA, Baptista TWF. A Política Nacional de Promoção da Saúde: texto e contexto de uma política. Saúde Debate [Internet]. 2015 [citado 2018 dez. 16];39(n.esp):91-104. Disponível em: http://www.scielo.br/scielo.php?pid=S0103-11042015000500091 \&script=sci_abstract\&tlng=pt

16. Conselho Regional de Enfermagem de São Paulo. Guia para construção de protocolos assistenciais de enfermagem [Internet]. São Paulo: COREN; 2017 [citado 2018 nov. 2]. Disponível em: https://portal.coren-sp.gov.br/sites/default/files/Protocolo-web.pdf

17. Gulini JEHMB, Nascimento ERP, Moritz RD, Rosa LM, Silveira NR, Vargas MAO. Intensive care unit team perception of palliative care: the discourse of the collective subject. Rev Esc Enferm USP. 2017;51:e03221. DOI: http://dx.doi.org/10.1590/S1980-220X20160 41703221

18. Manso MEG, Biffi ECA, Gerardi TJ. Prescrição inadequada de medicamentos a idosos portadores de doenças crônicas em um plano de saúde no município de São Paulo, Brasil. Rev Bras Geriatr Gerontol [Internet]. 2015 [citado 2018 nov. 2]:18(1):151-64. Disponível em: http://www.scielo.br/scielo.php?pid=S1809-98232015000100151\&script=sci_abstract\&tlng=pt

19. Manso MEG, Osti AV, Borrozino NF, Maresti LTP. Avaliação multidimensional do idoso: resultados em um grupo de indivíduos vinculados a uma operadora de planos de saúde. Kairós Gerontol [Internet]. 2018 [citado 2018 nov. 2];21(1):191-211. Disponível em: https://revistas. pucsp.br/kairos/article/view/38758/26308

20. Santos FPA, Acioli S, Machado JC, Souza MS, Rodrigues VP, Couto TA. Care practices of the Family Health Strategy team. J Nurs UFPE on line [Internet]. 2018 [cited 15 Dec 2018];12(1):36-43. Available from: https://periodicos.ufpe.br/revistas/revistaenfermagem/ article/ view/230589/25842

21. Tavares EL, Santos DM, Ferreira AA, Menezes MFG. Nutritional assessment for the elderly: modern challenges. Rev Bras Geriatr Gerontol [Internet]. 2015 [cited 2017 Dec 17];18(3):643-50. Available from: http://www.scielo.br/scielo.php?script=sci_arttext\&pid=S1809$98232015000300643 \& \operatorname{lng}=$ en \&nrm=iso\&tlng=en

22. Hooper L. Why, oh why, are so many older adults not drinking enough fluid? J Acad Nutr Diet. 2016;116(5):774-8. DOI: 10.1016/j. jand.2016.01.006

23. Hooper L, Bunn D, Jimoh FO, Fairweather-Tait SJ. Water-loss dehydration and aging. Mech Ageing Dev. 2014;136/137:50-8. DOI: 10.1016/j.mad.2013.11.009

24. Rocha LS, Beuter M, Neves ET, Leite MT, Brondani CM, Perlini NMOG. Self-care of elderly cancer patients undergoing outpatient treatment. Texto Contexto Enferm [Internet]. 2014 [cited 2018 Nov 2];23(1):29-37. Available from: http://www.scielo.br/scielo.php?script=sci_artte xt\&pid=S0104-07072014000100029

25. Couto R. Independência e autonomia de velhos que moram sozinhos. Memorialidades [Internet]. 2014 [citado 2018 nov. 2];10(19):119-26. Disponível em: http://periodicos.uesc.br/index.php/memorialidades/article/view/18 